\title{
Rede e Intersetorialidade na Atenção Psicossocial: Contextualizando o Papel do Ambulatório de Saúde Mental
}

\author{
Network and Intersectorality in \\ the Psychosocial Attention: Contextualizing \\ the Role of The Mental Health Clinic \\ Red e Intersectorialidad en la \\ Atención Psicosocial: \\ Contextualizando el Papel del \\ Ambulatorio de Salud Mental
}

Ana Kalliny Severo

Universidade Estadual de

Campinas

Magda Dimenstein

Universidade Federal do Rio

Grande do Norte
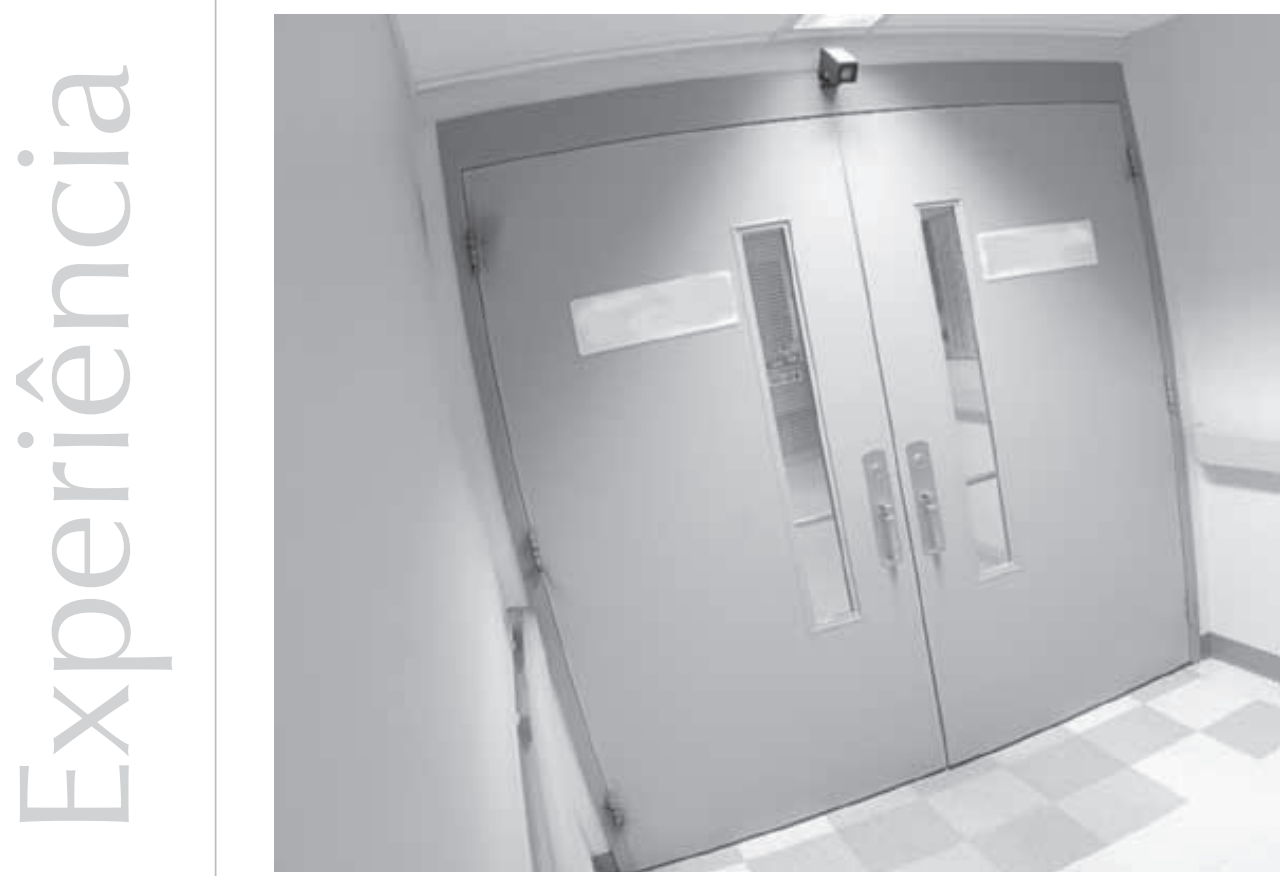
Resumo: A permanência e a função dos ambulatórios de saúde mental - ASM, que historicamente funcionaram articulados ao hospital psiquiátrico, precisam ser problematizadas no contexto atual de mudanças orientadas pela Estratégia de Atenção Psicossocial. O objetivo desta pesquisa foi conhecer e analisar o funcionamento de um ambulatório de saúde mental e sua articulação com a rede de serviços do Sistema Único de Saúde. A análise institucional de ênfase socioanalítica foi utilizada como perspectiva teórica e metodológica, e os procedimentos realizados foram: pesquisa bibliográfica e documental, análise dos registros do serviço, observação participante da rotina institucional e registros no diário de campo e rodas de conversa com os técnicos e usuários. O ASM atende 214 usuários que buscam principalmente assistência psiquiátrica e psicológica, direitos especiais, lazer e convívio social e cursos profissionalizantes. A principal dificuldade em relação à rede de atendimento apontada foi a desarticulação entre os serviços de saúde mental e a atenção básica. Para análise dos dados, destacamos dois analisadores principais: 1. as demandas atendidas no ambulatório de saúde mental e 2. a desarticulação saúde mental-atenção básica. As análises indicam a insuficiência dos dispositivos da rede, a necessidade de rever o dispositivo ambulatorial e os limites postos pela falta de políticas intersetoriais.

Palavras-chaves: Saúde mental. Atenção à saúde. Assistência à saúde. Serviços de saúde mental.

Abstract: The sustainability and function of outpatient mental health clinics, which have historically been part of the psychiatric hospital system, need to be considered in the context of the current changes driven by the Psychosocial Care Strategy. The objective of this research was to understand and analyze the operation of a mental health outpatient clinic and its link with the network of services of the National Health System. The methodological and theoretical basis used for the institutional analysis was the social-analytical perspective. The performed procedures were: bibliographic and documentary research, analysis of the record service, participant observation of the institutional routine and daily records in the field and conversation circles with the workers and users. The mental health clinic serves 214 users who seek mainly psychiatric and psychological care, special rights, leisure, social life and professional courses. The primary issue related to the service network was identified as the disconnection between mental health services and primary care. For data analysis, we list two points of argument: 1. the demands for services in the outpatient mental health care clinic and 2. disconnection mental health-primary care. The analyses indicated a failure of network care strategies, the need to revise the clinic protocols and the existence of care limitations due to a lack of sector integration policies.

Keywords: Mental health. Health attention. Health care. Mental health services.

Resumen: La permanencia y la función de los ambulatorios de salud mental - ASM, que históricamente han funcionado articulados al hospital psiquiátrico, necesitan ser ubicadas en los problemas del contexto actual de cambios orientados por la Estrategia de Atención Psicosocial. El objetivo de esta investigación ha sido el de analizar el funcionamiento de un ambulatorio de salud mental y su articulación con la red de servicios del Sistema Único de Salud. El análisis institucional de énfasis socio analítico ha sido llevado a cabo como perspectiva teórica y metodológica, y los procedimientos realizados han sido: investigación bibliográfica y documental, análisis de los registros del servicio, observación participante de la rutina institucional y registros en el diario de campo y ruedas de conversación con los técnicos y usuarios. El ASM alcanza 214 usuarios que buscan principalmente asistencia psiquiátrica y psicológica, derechos especiales, ocio y convivencia social y cursos capacitadores. La principal dificultad en relación a la red de atención señalada ha sido la desarticulación entre los servicios de salud mental y la atención básica. Para el análisis de los datos, destacamos dos analizadores principales: 1. las demandas atendidas en el ambulatorio de salud mental; y 2. la desarticulación salud mental / atención básica. Los análisis señalan la insuficiencia de los dispositivos de la red, la necesidad de revisión del dispositivo del ambulatorio y los límites impuestos por la falta de políticas intersectoriales.

Palabras clave: Salud mental. Atención a la salud. Prestación de Atención de Salud. Serviços de salud mental. 
A partir da implantação do Sistema Único de Saúde (SUS) no final da década de 1980, estruturou-se no País uma rede de serviços em nível crescente de complexidade norteada pelo ideal de justiça social (Cecilio, 1997). A reforma psiquiátrica está entrelaçada ao SUS e compartilha de seus princípios e diretrizes. Destacamos neste artigo a concepção ampliada de saúde, a perspectiva da atuação territorial, a intersetorialidade das políticas e o trabalho em rede. Tais elementos são aqueles que orientam a perspectiva da desinstitucionalização, bem como a Estratégia de Atenção Psicossocial (EAPs), pilares da política de saúde mental no País.

Embasada na reforma psiquiátrica, a implementação de uma nova lógica de cuidados aos portadores de transtornos mentais implica a superação do modo asilar e a implementação do modelo de atenção psicossocial. Concebido como Estratégia de Atenção Psicossocial/EAPs, tal modelo paradigmático vem evidenciando mudanças, avançando na construção de uma diversidade de dispositivos territoriais de atenção e cuidado. Dessa forma, a EAPs é uma lógica baseada na "integralidade das problemáticas de saúde e na ação territorializada sobre elas" (Yasui \& Costa-Rosa, 2008, p. 36).

Nesse contexto de produção de novas estratégias de acolhimento e cuidado, os dispositivos precisam ser constantemente revistos. Destacamos neste artigo a necessidade de problematização da permanência e da função dos ambulatórios de saúde mental/ASM no âmbito da reforma psiquiátrica brasileira, os quais funcionaram historicamente articulados ao hospital psiquiátrico. Para tanto, desenvolvemos um estudo ${ }^{1}$ cujo objetivo foi conhecer e analisar o funcionamento de um ASM e sua articulação com a rede de serviços do SUS.

\section{A rede de atenção psicossocial e os ambulatórios de saúde mental}

Os serviços públicos de saúde estão articulados em rede, em um modo de organização piramidal, em cuja base existe um conjunto de serviços responsáveis pela atenção primária, que funciona como porta de entrada dos usuários no sistema de saúde. Em um nível intermediário da pirâmide, situam-se os serviços de atenção de média complexidade e, no topo da pirâmide, estão os de alta complexidade, como os serviços hospitalares (Cecilio, 1997).

Entretanto, algumas críticas e reformulações têm sido feitas a esse modelo de rede. Passos e Barros (2004) criticam a organização piramidal dos serviços no SUS pelo fato de funcionarem com processos de verticalização de cima para baixo, ou vice-versa. Além disso, afirmam que toda rede deveria operar de um modo descentralizado, sem um centro de gerenciamento. Destacam que o Ministério da Saúde, recentemente, através da Política Nacional de Humanização, vem desenvolvendo uma proposta de rede para além de um conjunto de serviços, programas ou prescrições, mas que funciona como um conjunto de ações que transversalmente vão se articulando.

Barros (2003) tem alertado ainda que a rede de saúde se mostra como um conjunto de pontos fragilmente articulados e burocratizados. No caso da saúde mental, essa situação se agrava, pois, historicamente, sempre houve a fragmentação dos serviços de saúde mental da rede de saúde em geral, consolidando o modelo hospitalocêntrico. Isso gerou uma exclusão do portador de transtorno mental da rede de saúde geral bem como do meio social. Ademais, essa desarticulação tem produzido o que Barros denomina novas cronicidades: "1.'novas cronicidades' dos pacientes: ex- 
Santos (2007) aponta ainda a manutenção do ciclo criseinternação-altacrise-reinternação pela falta de uma política assistencial que delimitasse 0 funcionamento dos ambulatórios e pela falta de capacitação dos profissionais de saúde mental. pacientes, agora usuários (...), são postos como seres passivos frente ao desafio da produção de outra subjetividade. (...) 3. a cronicidade produzida pela inexistência ou fragilidade da rede de atenção em saúde: (...) que se verifica na existência desorganizada de várias portas de entrada (...) e uma falta de portas de saída" (Barros, 2003, pp. 204-205).

Destacamos que tais cronicidades se expressam através da retenção, por tempo indeterminado, dos usuários nos serviços de saúde mental, da dificuldade de produzir ações fora dos muros desses serviços, de construir portas de saída e de circulação na rede, de inventar novas relações e sociabilidades na comunidade e de espaço urbano para as pessoas que sofrem de transtornos mentais. É nessa perspectiva que questionamos o papel ocupado pelo ASM no contexto atual da reforma psiquiátrica: afinal, é um serviço que vem se apresentando como um facilitador de processos de cronificação?

Segundo a Portaria da Secretaria Nacional de Assistência à Saúde/Ministério da Saúde/ no 224/1992, o ambulatório, no âmbito da reforma psiquiátrica, deve seguir as diretrizes do SUS, podendo englobar unidades básicas/centros de saúde e ambulatórios especializados inseridos ou não em policlínicas. As atividades desenvolvidas devem concentrar-se nos atendimentos individuais, no atendimento grupal, nas visitas domiciliares e nas atividades comunitárias, especialmente na área de referência, sendo função do gestor local definir seu papel na rede de saúde.

O Ministério da Saúde informa que existem no País 862 serviços desse tipo, caracterizados atualmente pelo atendimento aos usuários menos graves (cerca de 9\% da população geral brasileira), pela baixa articulação com a rede de atenção em saúde, pela referência às consultas de Psicologia e psiquiatria, pela baixa resolutividade, com imensas listas de espera e com crises frequentes no funcionamento (Brasil, 2007). Pelas características apontadas anteriormente, existe a necessidade de aprofundar a discussão em torno das diretrizes de funcionamento dos ambulatórios, de sua função na rede de saúde mental e de sua inserção na Estratégia de Atenção Psicossocial.

O surgimento dos ambulatórios especializados em saúde mental no Brasil ocorreu na década de 20, com a implantação da Política Assistencial de Higiene Mental, objetivando prevenir transtornos mentais na população. Nessa estratégia, a psiquiatria atuaria mais próximo da comunidade, na perspectiva de normalização social, da eugenia, do exercício de uma vigília, do controle dos imigrantes e dos matrimônios, na tentativa de evitar uma deterioração hereditária (Lougon, 2006). Essa lógica perdurou até a década de 80 , quando foi elaborado o Plano de Reorientação da Assistência Psiquiátrica pelo Conselho Consultivo da Administração de Saúde Previdenciária (CONASP), através do qual ocorreu um grande investimento do Estado no sistema de atendimento público, priorizando a reforma dos hospitais psiquiátricos e a expansão de uma rede ambulatorial extramuros, na busca da reinterpretação do fenômeno do adoecimento mental.

Entretanto, essa reforma assistencial não obteve êxito. Goulart (2006) aponta, em estudo feito em Minas Gerais, o aumento da procura da população pelos ambulatórios de saúde mental para adquirir receitas médicas e psicotrópicos e a distorção da demanda, excluindo os casos graves e os problemas de resolutividade (psicologização e medicalização da mesma). Santos (2007) aponta ainda a manutenção do ciclo crise-internação-altacrise-reinternação pela falta de uma política assistencial que delimitasse o funcionamento dos ambulatórios e pela falta de capacitação dos profissionais de saúde mental. De acordo com esse autor, "a proposta de ambulatorização não questionava os efeitos 
negativos do manicômio: a cronificação, a estigmatização do sujeito internado, a hierarquização entre os membros da equipe, o imperativo do saber médico" ( $p$. 67). Desse modo, vemos que o processo de ambulatorização da saúde mental ocorrido no País resultou na cronificação da população assistida, atraindo novos pacientes e elevando o consumo de psicotrópicos. Tais consequências são sentidas até hoje, aspecto que consideramos problemático no processo de reforma psiquiátrica, já que esta busca operar a mudança paradigmática do modo asilar hegemônico para o modo psicossocial.

Costa-Rosa (2000) designa as mudanças da reforma psiquiátrica a partir da distinção de dois modelos paradigmáticos, sendo um caracterizado como modo asilar e o outro como psicossocial. O autor explica que esses dois modelos só podem ser alternativos um ao outro se considerados contraditórios, ou seja, se suas práticas expressarem parâmetros basilares ou paradigmas opostos. Segundo essa perspectiva, afirmar que o modelo ambulatorial e multiprofissional é diferente do médico-centrado e hospitalocêntrico não os define como alternativos e/ou contraditórios. O primeiro modelo pode comportar características do segundo se suas práticas se fundamentarem, por exemplo, no depósito de expectativas na medicação, no saber psiquiátrico, no recurso às internações psiquiátricas, etc. Nesse sentido, Alves e Guljor (2004) explicitam que os ambulatórios de saúde mental não constituem, por definição, um serviço ultrapassado, mas um serviço aberto que, para ser potente, necessita assumir, em suas práticas, características de um serviço de atenção psicossocial.

É necessário ressaltar ainda que o paradigma psicossocial situe a saúde mental na saúde coletiva, reconhecendo as necessidades específicas de cada grupo, e adota práticas de cuidado no sentido da prevenção e da promoção da saúde, ampliando assim o horizonte das intervenções e ultrapassando as separações artificiais das políticas setoriais (Vasconcelos, 2009). Nessa perspectiva, a intersetorialidade e a territorialidade devem ser norteadores das práticas e do planejamento em saúde.

No campo da reforma psiquiátrica, devem-se ampliar as intervenções além de serviços ou equipamentos de saúde para que se efetive a reinserção social de pessoas portadoras de transtornos mentais, que foram secularmente estigmatizadas e excluídas socialmente, sendo incluídos outros estabelecimentos, como associações, cooperativas e variados espaços das cidades. Desse modo, a partir das articulações produzidas para responder às demandas de cuidados, um dispositivo de saúde mental assume funções sociais em um determinado contexto.

Segundo Costa-Rosa, é necessário avaliar as funções sociais dos dispositivos de saúde mental. No caso dos ambulatórios de saúde mental, é necessário analisar sua função positiva explícita no discurso, como a produção de saúde, o atendimento multiprofissional, a reinserção social, etc., como também perceber sua função negativa, através da análise das lacunas presentes no discurso e nas práticas nem sempre transparentes e acessíveis. Para isso, tornase imprescindível examinar a distinção dos modelos hospitalocêntrico e medicalizador e do paradigma da atenção psicossocial nas formas como esses estabelecimentos de atendimento se relacionam com sua clientela (Costa-Rosa, 2000).

No modo asilar, os estabelecimentos são lócus depositários para onde se enviam todos os problemáticos para que sejam tratados e devolvidos ao sistema social tal como eram antes. Nesse sentido, esses estabelecimentos tendem a funcionar como "espaços depositários de tutoria e, de modo mais amplo, pode-se considerá-la como 
agenciadora de suprimentos, diante de uma clientela considerada carente" (Costa-Rosa, 2000, p. 161). Já na atenção psicossocial, os estabelecimentos caracterizam-se por serem espaços de interlocução entre os sujeitos participantes, devendo ser capazes de funcionar para além de depositários e de tutelares, discriminando a dimensão da demanda social e encaminhando-a para outros espaços de encomenda, exercendo um estreitamento da faixa do tratável e recorrendo a uma série de encomendas a partir da análise da demanda social que lhes chega (Costa-Rosa, 2000).

Os ambulatórios de saúde mental vêm auxiliando na manutenção do primeiro modelo por centrarem-se muito mais nos especialismos e por adotarem um modelo baseado no saber psiquiátrico, que seleciona a demanda para os hospitais psiquiátricos (Peixoto, 2007). Atualmente, apesar de estarem inseridos em uma política assistencial fundamentada nos princípios da reforma psiquiátrica e nos princípios do SUS, os ASMs ainda se mostram como referências para inúmeras consultas em psiquiatria e Psicologia, apresentando problemas como a pouca resolutividade e a baixa articulação com a rede de saúde mental (Brasil, 2006). A partir da percepção de que os ambulatórios de saúde mental estão historicamente ligados a uma lógica de atendimento centrada no saber psiquiátrico, em que este tinha na rede uma função complementar e não substitutiva aos hospitais psiquiátricos, pretendemos analisar as características do ambulatório de saúde mental e sua pertinência frente ao modelo de reforma psiquiátrica atual e da perspectiva da rede adotada pela EAPs, analisando as demandas sociais que Ihe chegam e as encomendas que esse estabelecimento tem feito para responder a essa demanda.

\section{O percurso da pesquisa}

Este estudo foi orientado prioritariamente pela perspectiva qualitativa de pesquisa, por privilegiar os sentidos e os afetos dos movimentos de uma comunidade ou grupo social, percebendo-os "nas polêmicas, nos desvios, nas ações que fazem diferença, frente ao hegemônico, que abre espaço a imprevisibilidades" (Rocha, 2006, p. 171). Entretanto, em alguns momentos, adotamos alguns procedimentos da pesquisa quantitativa, na análise dos índices dos registros do serviço.

A análise institucional, mais precisamente na ênfase socioanalítica, foi adotada como perspectiva teórico-metodológica. Nessa perspectiva, a análise da encomenda e da demanda, a intensificação da autogestão pelo coletivo-cliente, a explicitação das transversalidades e a elucidação dos analisadores se tornam imprescindíveis (Rodrigues, 2004). Norteamos a investigação a partir desses conceitos-procedimentoschave, com a finalidade de construir um movimento de pesquisa-intervenção. Com base nisso, ao mapear as forças que compõem a realidade pesquisada, buscamos elucidar as instituições e seu funcionamento através de duas dimensões: instituinte e instituída. Essas dimensões acabam por dar processualidade, fazendo emergir o novo e rompendo a lógica de funcionamento enrijecida (dimensão instituinte), ou mesmo perpetuando práticas, modos de pensar, de viver e de funcionar (dimensão instituída). Essa processualidade se evidencia em nível organizacional, através das dimensões organizante e organizada. A primeira se caracteriza por ser transformadora, por refletir sobre as organizações, podendo trazer novas lógicas de funcionamento. Já a segunda diz respeito ao que se reproduz, sendo ilustrada pelos organogramas que representam a cristalização do funcionamento de uma determinada organização. 
Escolhemos como espaço de pesquisa um serviço ambulatorial especializado em saúde mental de um Município nordestino destinado a receber usuários egressos dos Centros de Atenção Psicossocial II. O ASM tenta funcionar como porta de saída da rede substitutiva para esses usuários. Entretanto, isso tem se mostrado problemático, pois os usuários não conseguem sair da rede especializada em saúde mental e aderir a um tratamento em saúde mental na rede básica de saúde.

O ASM funciona de segunda a sexta-feira, das oito às doze horas, nas dependências de um centro clínico da cidade. Seu cronograma de atividades estava distribuído em atividades grupais, e abrangia oficinas de artes e artesanato, grupos terapêuticos, consultas psiquiátricas individuais e escuta psicológica. Outras atividades, como grupos de dança, passeios e sessões de cinema, eram inseridas na programação cotidiana do serviço conforme a demanda dos usuários. A equipe do ambulatório era composta por 10 técnicos, sendo 3 psicólogas, 1 assistente social, 1 filósofa, 1 arte-educadora, 1 auxiliar de enfermagem e 3 psiquiatras. Existiam 214 usuários cadastrados no serviço.

Após a permissão dos técnicos e usuários do serviço para a realização da pesquisa, realizamos três meses de observação das atividades cotidianas do ASM, durante quatro horas diárias, ao longo de trinta e seis dias de observação. As observações centraram-se principalmente na sala de espera e em uma atividade de grupo denominada Bom-dia, caracterizada como espaço de conversa grupal, da qual todos os usuários participam, sob a coordenação de um dos técnicos do serviço. Realizamos ainda análise dos registros do serviço que continham dados sobre os usuários (tempo no serviço, dados socioeconômicos e familiares, circulação pela rede de serviços de saúde, projeto terapêutico, etc.). Além disso, realizamos 5 rodas de conversa com 7 técnicos dos 10 profissionais, e 1 roda de conversa com 9 usuários. As rodas de conversa são utilizadas nas metodologias participativas e buscam sensibilizar e mobilizar os participantes a refletir acerca de sua relação com o mundo, com o trabalho, com seu projeto de vida, etc. (Afonso \& Abade, 2008). As temáticas das rodas de conversa não foram delimitadas a priori, mas surgiram a partir da dinâmica existente nas rodas. No início de cada uma delas, havia o processo de restituição daquilo que estávamos refletindo acerca desse campo e dos levantamentos feitos a partir dos dados.

Identificamos alguns analisadores que subsidiaram reflexões junto ao coletivo pesquisado. Um analisador refere-se a um fenômeno emergente de uma série de forças contraditórias apresentadas na realidade (Baremblitt, 1992). Desse modo, destacamos dois analisadores principais e construímos dois eixos de análise que serão focos de discussão: a demanda atendida no ASM e a desarticulação da saúde mental com a atenção básica. Tais eixos foram articulados na perspectiva de problematizar o lugar do ASM no contexto da Estratégia de Atenção Psicossocial.

\section{Resultados e discussão}

\section{Demanda atendida}

No serviço ambulatorial estudado, encontramos cinco aspectos principais em relação ao atendimento da demanda, a saber: a demanda por psicotrópicos e consultas psiquiátricas, a busca pela escuta psicológica, a demanda por direitos especiais e a demanda por convivência e circulação social. A seguir, detalharemos cada uma delas.

a) A demanda por psicotrópicos e pela assistência psiquiátrica:

Sobre os usuários, haviam 214 cadastrados no serviço, e todos faziam uso de consultas psiquiátricas. Destes, 26\% de usuários $(n=55)$ 
faziam uso de mais de uma atividade (Bomdia, grupos terapêuticos, oficinas, etc.) e $63 \%$ $(n=135)$ frequentavam apenas as consultas psiquiátricas.

Percebemos que um reduzido número de usuários participava de outras atividades. Diante desse quadro e do fato da maior parte ser atendida há mais de cinco anos no serviço (77\%), surgiu o questionamento: por que um serviço de saúde mental que se propõe a funcionar no modelo de atenção psicossocial ainda tem como atividade principal a administração de psicotrópicos? Segundo Bezerra Jr. (2007), diversas questões estão envolvidas nessa problemática, e uma delas refere-se aos medicamentos, doenças e representações distintas entre a população atendida e os técnicos de saúde mental. A demanda da população por medicamentos nos ambulatórios de saúde mental está associada ao modo como a população concebe o adoecimento, atribuindo-lhe causas de ordem somática. A concepção de que a localização da doença está no corpo do usuário torna o medicamento imprescindível como intervenção terapêutica. O conhecimento e a prática médica, como sabemos, ainda mantêm um lugar de privilégio entre a população de modo geral. Além disso, o desenvolvimento dos psicotrópicos, principalmente depois da Segunda Guerra Mundial, quando as experiências de reformas psiquiátricas no mundo buscaram a saída das pessoas com trantornos mentais dos hospitais psiquiátricos, o largo uso dos neurolépticos, que têm a capacidade de reduzir os sintomas psicóticos, permitiu ao psiquiatra manter o seu mandato social sobre a loucura, "agindo como embaixatriz social das normas dominantes na sociedade" (Silva Filho, 2007, p. 96).

Destacamos, assim, que a grande demanda por consultas e psicotrópicos, bem como a preocupação com a desassistência em psiquiatria por parte dos técnicos e usuários quando se fala em alta, indica a predominância do modelo médico no ASM. Essa lógica alimenta os problemas e os torna crônicos. Em uma das rodas de conversa, os profissionais falam do medo da desassistência psiquiátrica diante de crises, com a falta de dispositivos substitutivos como o CAPS tipo III e de leitos psiquiátricos em hospitais gerais na cidade. O CAPS III funciona 24 horas e tem leitos para internações de curta duração. Já os CAPS II funcionam de segunda a sexta, nos turnos diurnos, não possuindo estrutura física e equipe para atender às crises fora desses horários. Em outras palavras, são poucas as iniciativas de mudança observadas na lógica de cronicidade dos problemas na implementação de dispositivos substitutivos na rede, que deveriam começar pela oferta de novas modalidades de cuidado, que implicariam, por fim, a mudança de concepção e de valor atribuído à loucura e ao aparato psiquiátrico. Isso quer dizer que o foco nas consultas e na prescrição de medicamentos observados no ASM confirma a força do paradigma racionalista psiquiátrico, que toma a loucura como objeto de sua intervenção, sendo, "portanto, um sistema de ação que intervém em relação a um problema dado (a doença) para perseguir uma solução racional, tendencialmente ótima (a cura)" (Rotelli, Leonardis, \& Mauri, 2001, p. 26).

\section{b) A demanda por escuta psicológica:}

Essa demanda tem ocupado também um lugar central no ambulatório, através da solicitação dos usuários por grupos terapêuticos e por uma escuta clínica individualizada junto às psicólogas do serviço. Os técnicos e os usuários atribuem papel importante a essa função, na medida em que aquelas atendem as urgências subjetivas (no dizer de uma psicóloga), e os usuários buscam uma escuta psicológica individualizada, por não querer expor alguns problemas diante de outros usuários. Isso foi observado principalmente em uma roda de conversa com os usuários. 
A lógica dos atendimentos individuais, a predominância desse modelo de escuta terapêutica que privilegia a vivência subjetiva e oferece psicoterapia, em detrimento das práticas mais participativas, comunitárias, grupais, pode estar indicando a necessidade de se rever esse tipo de oferta no serviço ambulatorial, a fim de se propor estratégias mais condizentes com a EAPs, buscando uma organização do trabalho no território de referência dos usuários, extramuros, em rede, de modo a aproximar-se da realidade e das necessidades concretas dos usuários atendidos (Bezerra Jr., 2007). Desse modo, percebemos que a predominância do modelo terapêutico psicoterápico, aliado ao medicamentoso, centra suas intervenções no indivíduo, mantendo uma lógica de dependência do usuário em relação aos saberes médico e psicológico e depositando nestes grandes expectativas na resolução dos seus problemas. Isso é percebido quando observamos a reduzida participação da família (36\%) nas atividades do serviço pesquisado, e, quando existe, é por solicitação da assistente social que havia iniciado um grupo de família. Ao contrário disso, estratégias de cuidado que englobassem recursos e grupos comunitários, que se tornassem redes de suporte social para os usuários, poderiam garantir promoção de modos de vida menos dependentes em relação ao serviço para os usuários.

\section{c) A demanda por direitos especiais:}

Outras solicitações frequentes são os atestados médicos com a finalidade de obtenção de pensões através da perícia médica e da aposentadoria, além de solicitações referentes à carteira de gratuidade destinada ao sistema de transporte público da STTU (Secretaria Municipal de Transporte e Trânsito Urbano), assuntos tratados principalmente com a assistente social do serviço. Em conversa com uma das usuárias do serviço, ela me narra sua experiência, dizendo que havia passado um ano no CAPS II e que havia ido para o ASM há cinco anos. Eu the perguntei se ela não tinha vontade de sair dali. Ela disse que poderia perder o benefício, e como seria? Disse que sua patroa não a queria mais, e não conseguia arranjar trabalho do seu jeito peculiar de fazer as coisas, condizente com suas possibilidades. Complementa ainda que estava pela perícia, e mencionou o fato de o juiz querer retirar o benefício que recebia há dois anos.

As pensões obtidas através do INSS (Instituto Nacional do Seguro Social) são destinadas aos usuários que comprovem incapacidade laborativa e que estejam em tratamento em algum serviço de saúde mental. Segundo as Diretrizes de Conduta Médico-pericial em Transtornos Mentais, para a concessão do benefício, faz-se necessária a comprovação do tratamento psiquiátrico (Brasil, 2007).

O fato de esses usuários estarem vinculados ao serviço ambulatorial é um facilitador para a obtenção desses atestados e para a comprovação das suas incapacidades de trabalhar (termo usado por usuário), permitindo, assim, que eles permaneçam pela perícia do INSS recebendo o benefício e também que obtenham, à custa de muitas dificuldades, a carteira da STTU. Mas, há algum risco nessa prática? Isso indica avanços em relação ao modelo tradicional de ambulatório e à produção de práticas mais sintonizadas com EAPs voltadas para as realidades concretas dos usuários, para a construção de alternativas de vida, de sociabilidade, de invenção de saúde? Podemos dizer que sim, mas isso traz consigo desafios, pois as questões de reivindicação por direitos especiais das pessoas com transtornos mentais, ao mesmo tempo em que beneficia os usuários, podem reforçar seu estatuto de tutelado e o poder do diagnóstico (psiquiátrico, psicológico, social) como critério de inclusão nos direitos especiais (Brasil, 2001). E ainda, esse tipo de reivindicação parte da busca da restituição da cidadania às pessoas com transtornos mentais, a partir da proposta de reabilitação social que se insere na reforma psiquiátrica brasileira. 
A reabilitação social, porém, ao pautar-se na perspectiva da cidadania, acaba por ser atravessada por contradições que se materializam nas novas práticas. As propostas de curatela, de trabalho protegido, da ideia de cidadania entre iguais e de sociedade inclusiva refletem questões como o limite versus a autonomia das pessoas portadoras de transtornos mentais, da igualdade e do direito à diferença, etc., contradições que aparecem fortemente no cotidiano do ambulatório e que necessitam ser refletidas junto aos usuários, principalmente no que se refere ao termo incapacidade, já que este é determinante para a obtenção dos benefícios.

A busca de reabilitação comporta, na prática dos serviços substitutivos, duas concepções de cidadania que podem favorecer ora processos de normalização social, ora de reconstrução de modos variados de se conviver com a diferença. O discurso da reinserção e da reabilitação psicossocial pode estar ancorado na concepção de incapacidades inerentes ao adoecimento mental e, portanto, da necessidade de recuperação daquilo que falta ao louco para que ele possa atender as expectativas de ser racional, consciente, normalizado, sujeito esse idealizado a partir do projeto da modernidade (Venturini, Galassi, Roda, \& Sérgio, 2003).

A concessão de benefícios sociais, portanto, poderia vir acompanhada de discussões sobre o projeto terapêutico singular, por exemplo. Entretanto, essa dissociação reforça ainda a dependência dos usuários em relação ao serviço, não se conseguindo alcançar sua efetiva reinserção no meio social. Nos dados pesquisados no cadastro do serviço, percebemos que os projetos terapêuticos predominantes são constituídos pelas consultas psiquiátricas e que as atividades projetadas coincidem com as mesmas atividades já desenvolvidas pelos usuários no serviço, sem nenhuma discussão para redefinição desses projetos. A busca por direitos especiais é um modo de sobrevivência dos usuários em um contexto de políticas públicas precárias, e isso precisa ser considerado nos serviços substitutivos. Dimenstein e Liberato (2009) ressaltam que um dos grandes desafios para a política em saúde mental diz respeito à falta de equipamentos sociais decorrentes, principalmente, do colapso das políticas públicas de bem-estar social, pois estas disponibilizariam a todos os cidadãos acesso a bens e serviços tal como é considerado seu direito.

d) A busca por convivência social e por cursos profissionalizantes:

O serviço ambulatorial é utilizado pelos usuários ainda como espaço de convivência, circulação e construção de amizades, através de festas comemorativas, passeios e discussões acerca de suas dificuldades cotidianas. Em uma das rodas de conversa com os usuários, foi dito que mediante a alta, por exemplo, eles sentiriam falta de frequentar o serviço devido às conversas com as psicólogas, as oficinas e os passeios. Um deles comenta que perderia seu local de lazer: Deus me livre de sair de lá. É onde eu me distraio...O único canto que eu saio é pra lá" (usuário).

Cotidianamente, todos os usuários que vão ao serviço trocam experiências acerca de suas atividades e das inúmeras dificuldades em relação à família, aos benefícios, à falta de medicação, etc. Os usuários se envolvem, fornecendo conselhos e contando suas experiências pessoais, tentando auxiliar no cuidado aos outros. Isso demonstra que o ASM tem a potencialidade de constituir um espaço vivo, de trocas sociais, de reprodução social dos usuários. Nesse sentido, o ASM funciona, no que se refere à atenção psicossocial, como lócus destinado a acolher o sofrimento vivido cotidianamente pelas pessoas.

A mobilização do Pastoril (dança popular característica no Nordeste) existente no ASM é exemplo de um importante dispositivo 
terapêutico que modificou o cotidiano e mobilizou diversos atores sociais. Nessa iniciativa, mudanças ocorreram no sentido de incluir a participação de outros técnicos do centro clínico no qual esse ASM se insere, e de outras pessoas da comunidade, que auxiliaram na confecção das roupas para o grupo de dança além de convites de apresentações em outros grupos e espaços sociais da cidade. Essa iniciativa revela que a intersetorialidade, através da articulação saúde mental-cultura, é muito potente na construção de parcerias com outros grupos sociais na conquista de outro lugar para a loucura no âmbito social. Serviços e políticas públicas norteados pela atenção psicossocial devem priorizar a intersetorialidade como uma estratégia fundamental na construção de projetos de saúde, de solidariedade e de participação social, tornando os sujeitos ativos na produção de saúde.

Além disso, consideramos que esses momentos de encontro entre usuários são uma importante estratégia de ajuda mútua, uma forma de fortalecimento pessoal e coletivo. O grupo de ajuda mútua diz respeito às trocas de experiência, de ajuda emocional e de reflexão em torno das diferentes estratégias para superar os problemas comuns (Vasconcelos, 2007). Acreditamos que o ambulatório, ao servir como espaço de convivência e de troca de experiência entre os usuários, pode ser um importante veículo de empoderamento. Vasconcelos define o empowerment, nomenclatura usada na literatura anglo-saxônica, ou empoderamento, como importante abordagem para a obtenção de mudanças nos modos de distribuição das relações de poder, buscando o "fortalecimento do poder, participação e da auto-organização dos usuários e familiares de serviços públicos, nos planos pessoal, interpessoal, grupal, institucional e na sociedade em geral" (2007, p. 175).
Esse mesmo autor afirma que o uso da abordagem do empoderamento pelos usuários e familiares, em nível internacional, principalmente em países anglo-saxônicos e no norte da Europa, tem obtido avanços no que se refere à estruturação do movimento protagonista, crítico e inovador, que se torna agente no movimento da reforma psiquiátrica.

\section{A desarticulação saúde mental- atenção básica}

Seguindo as recomendações do Ministério da Saúde para os gestores, a organização da rede de saúde mental para uma cidade com mais de 700 mil habitantes deveria se dar a partir de uma rede assistencial composta por CAPS II, CAPS III, CAPSad, CAPSi, rede básica com ações em saúde mental e capacitação para o SAMU. Entretanto, segundo a Secretaria de Saúde, a rede de saúde mental local está distribuída em 8 serviços substitutivos, realizados em 2 Centros de Atenção Psicossocial tipo II, 2 ambulatórios de saúde mental (Ambulatório de Prevenção e Tratamento do Tabagismo, Alcoolismo e outras Drogadições - APTAD e outro para atendimento de transtornos psiquiátricos gerais), 2 Centros de Atenção Psicossocial tipo AD, 1 Centro de Atenção Psicossocial tipo i (atendimento infanto-juvenil) e 1 residência terapêutica (egressos de longas internações e sem vínculos familiares) (Secretaria Municipal de Saúde, 2008).

Apesar da existência desses serviços substitutivos, percebemos que eles são insuficientes numericamente para atender à população, e os três hospitais psiquiátricos da cidade ainda continuam sendo referência no atendimento em saúde mental. O lugar que o serviço ambulatorial estudado tem tido na rede de saúde mental é de acolher, dentro da rede especializada, os usuários que receberam alta dos CAPS II e, atualmente, apresenta-se 
como mais um serviço que não absorve a demanda pela superlotação. A maioria dos usuários do ASM foi encaminhada pelos CAPS II ou pelo hospital-dia (49\%). Desse modo, durante as observações, um dos participantes afirma que o ASM representa o fim de carreira do usuário da saúde mental. Pensando nisso, em uma das rodas de conversa com os profissionais, eles apontaram principalmente a desarticulação entre os serviços substitutivos e o sistema básico de saúde na rede de saúde mental como causa desse problema.

Uma saída para isso seria o apoio matricial nas unidades básicas de saúde. Segundo a Secretaria Municipal de Saúde (2006), existe a tentativa de implementação do apoio matricial em seis unidades básicas de saúde, mas algumas pesquisas têm indicado a desarticulação entre os serviços substitutivos e a atenção básica. Além disso, o fato de o ambulatório constituir o lugar de fim de carreira para os usuários da saúde mental indica que essa proposta não tem funcionado na prática. Os casos atendidos nos serviços substitutivos não têm encontrado uma efetiva co-responsabilização entre as equipes de saúde mental e as unidades básicas de saúde. O que tem prevalecido é a perspectiva médica tradicional, em que os problemas em saúde mental devem ser atendidos por um serviço especializado, já que os usuários saem dos CAPS e necessitam de outro serviço com a mesma competência especializada, como o ambulatório, para serem atendidos.

Entretanto, uma psicóloga realizava matriciamento em uma unidade básica de saúde, e essa experiência de coresponsabilização no atendimento aos usuários atendidos apenas pela unidade de saúde teve bastante êxito. Em um dos casos relatados por uma das psicólogas, o de uma jovem que sofria de depressão pós-parto, o agente comunitário de saúde dialogou com a equipe da unidade, que entrou em contato com o ASM. A psicóloga se dispôs a fazer reuniões com a equipe da unidade, e executaram visitas domiciliares junto ao agente comunitário de saúde, o que possibilitou o acompanhamento da jovem pela equipe da unidade e a melhora da usuária atendida.

Nesse trabalho de matriciamento e de coresponsabilização, foi essencial para o êxito da iniciativa a disponibilidade do técnico do serviço ambulatorial em ir até a unidade de saúde e a confiança da equipe da unidade em recorrer ao ASM. Desse modo, percebemos que é necessário investir na rede de suporte entre profissionais como fator essencial na co-responsabilização pelos casos em saúde, formando uma rede aquecida. Entretanto, dificuldades maiores são postas quando se fala dessas práticas de matriciamento. Os técnicos, em algumas rodas de conversa, apontam como dificuldade o despreparo das equipes da atenção básica em lidar com essa demanda. Essa realidade é encontrada nacionalmente, havendo uma forte resistência das equipes dos CAPS em dar a retaguarda para a atenção básica, pois essa estratégia é vista como mais uma tarefa a ser inserida na rotina desses serviços (Dimenstein \& Liberato, 2009). Enquanto isso, os técnicos da atenção básica não se sentem capacitados para atender à demanda de saúde mental na atenção primária, ficando desassistida grande parte da população.

Os usuários que entram nos serviços substitutivos na cidade acabam sendo atendidos, no campo da saúde mental, apenas em serviços especializados ou nos hospitais psiquiátricos. A dificuldade de articulação entre os serviços substitutivos e as intervenções fragmentadas e pouco flexíveis auxiliam na manutenção da circulação dos usuários no circuito psiquiátrico, onde as estruturas de atendimento comunitário vêm complementar a internação psiquiátrica, de modo que a segregação anteriormente 
estática e restrita ao hospital psiquiátrico passa a ser dinâmica pela circulação por serviços especializados, com intervenções pontuais e fragmentadas (Rotelli, Leonardis, \& Mauri, 2001). É necessário que os serviços comunitários, tal como os CAPS, alterem seu modelo cultural de referência para modificar os modelos de intervenção (Dell'Acqua \& Mezzina, 2005). Nesse sentido, os serviços substitutivos, ao adotar a proposta de desinstitucionalização, deveriam substituir a relação centrada na doença, que tem como centro o hospital, para ter como referência a reprodução social do usuário e o território. Desse modo, poderiam atender o usuário, objetivando a reconstrução da sua vida e dos laços sociais diversos.

Amarante aponta a importância da inserção da saúde mental na saúde da família, pelo fato de a Estratégia de Saúde da Família (ESF) ter como foco a família e tentar reverter o modelo assistencial predominantemente biomédico, cujo centro é a doença e o tratamento, para investir na promoção da saúde e na defesa da vida. A ESF surgiu em 1994, sob a denominação Programa de Saúde da Família. Ela é composta por 1 médico generalista, 1 enfermeiro, 1 auxiliar de enfermagem e por 4 a 6 agentes de saúde, sendo cada equipe responsável por 800 famílias (Amarante, 2007). Esse autor afirma ainda que a ESF pode auxiliar na desmedicalização, negando a compreensão de que todos os problemas de uma comunidade são médico-sanitários e dispensando ainda muitos encaminhamentos aos níveis mais complexos da atenção, "não valorizando ou estimulando excessivamente a 'carreira do doente' na pessoa que vive a experiência da doença" (p. 95).

A ideia de complexidade que rege a atual organização piramidal da rede, na qual a atenção básica é responsável por intervenções mais simplificadas, é invertida no caso da saúde mental (Amarante, 2007). No hospital, as respostas aos problemas de saúde mental são padronizadas e massificadas, no nível primário da atenção; essas respostas devem ser mais complexas, englobando a família, a vizinhança e os atores sociais no território em que vivem. Por isso, investir em uma articulação entre a rede de saúde mental e a atenção básica é indispensável para a efetivação da reforma psiquiátrica.

Para a demanda atendida no ambulatório, a inserção da saúde mental na atenção básica seria um recurso muito produtivo para desconstruir a carreira institucional vivenciada pelos usuários, além de ser possibilidade privilegiada de atendimento, seguindo uma perspectiva de integralidade para a imensa maioria dos usuários que são atendidos apenas para consultas psiquiátricas no ambulatório. As possibilidades de coresponsabilização aconteceriam na medida em que a lógica de trabalho, centrada no ASM, englobasse contatos frequentes com a atenção básica, buscando, junto com o usuário e as equipes de Saúde da Família, recursos territoriais e comunitários, alargando assim a rede de suporte social e de cuidado. Entretanto, isso não se efetiva, e o serviço atende usuários de todos os distritos sanitários da cidade, o que aumenta ainda mais o quadro de fragmentação entre os serviços assistenciais.

\section{Considerações finais}

Analisando a oferta e o modo como o ambulatório de saúde mental vem operando, percebemos que ele vem ocultando processos de cronificação, ou de cronicidade, em três diferentes níveis: 1. ao aglutinar funções que deveriam estar sendo realizadas por outros dispositivos da rede de saúde mental, como os centros de convivência e cultura, as cooperativas de trabalho, os serviços de atenção à crise tipo CAPS III, bem como pela rede de saúde do SUS, especialmente na atenção básica via ESF; 2. nos CAPS, que não vêm conseguindo produzir ações territoriais e ser ordenadores da rede e 3 . nos processos de gestão e trabalho em saúde mental. 
A diversidade de demandas atendidas no serviço acaba por estimular a dependência dos usuários em relação aos serviços substitutivos, produzindo a cronificação e um trânsito restrito no circuito especial (rede especializada de serviços de saúde mental - domicílio). Algumas propostas de desinstitucionalização, ao resultarem na cronificação, apontam a tentativa fracassada de liberar a psiquiatria de sua relação histórica com a justiça e o controle social, enfatizando sua vocação terapêutica. As diversas demandas atendidas e concentradas no ambulatório e o quadro de desarticulação da rede de saúde mental com a atenção básica e com outros dispositivos sociais de cuidado indicam a prevalência do paradigma hospitalocêntrico, asilar. A cronificação na rede evidenciada pela falta de alternativas diversificadas que poderiam funcionar como dispositivos de encomenda para responder a essas demandas acaba por reafirmar a psiquiatrização da demanda social. Isso indica a necessidade de investir em outras instâncias de encomendas sociais, para que não se mantenha um processo de repetição, cujo tratamento se centra na medicação e na escuta psicológica, pensando em uma lógica mais coerente com o paradigma psicossocial, na tentativa de ampliar a visão referente aos problemas de saúde dos usuários, afirmando a sua desospitalização, desmedicalização e envolvimento subjetivo e sociocultural.

No quadro mencionado, percebemos a cronificação presente no estabelecimento ambulatorial e na rede de saúde mental, a dificuldade de trabalho em rede e a necessidade de realizar iniciativas intersetoriais. É necessário atualmente considerar esse modelo ambulatorial um modelo transitório que guarda resquícios, em seu funcionamento, de complementaridade ao hospital psiquiátrico, e não de substituição, e que é incoerente com o paradigma psicossocial. Essas estruturas devem assumir outra função na rede de saúde, a partir da sua dimensão instituinte já observável no seu cotidiano, através do fornecimento de um espaço de acolhimento, de convívio e de ajuda mútua entre os usuários e das atuações relativas ao apoio matricial. Concordamos com Yasui e Costa-Rosa quando afirmam que os ambulatórios de saúde mental surgiram com a proposta preventivista, e que a sua persistência ao conviver com um CAPS em um mesmo território acaba por reproduzir o efeito complementar e não substitutivo ao hospital psiquiátrico, e, portanto, contraditório à lógica da atenção psicossocial.

Adotar uma perspectiva de atenção psicossocial permitirá, por exemplo:
re-configurar os CAPS como instâncias aptas a responder à especificidade das demandas que Ihes são atribuídas: demandas específicas de sofrimento psíquico com exigências de intensidade variada, que vão da exigência máxima que define o CAPS atual até as intensidades variadas que definem atualmente, de modo geral, o ambulatório (Yasui \& Costa-Rosa, 2008, p. 36)

Desse modo, é necessário investir no CAPS, na sua articulação com a atenção básica e com o território e investir na própria atenção básica como estratégia primordial para o acolhimento e a promoção de ações em saúde mental. Dimenstein e Liberato (2009) ressaltam que

A cronificação provocada pela falta de articulação de diferentes serviços e apoios sociais, culturais e assistenciais produz efeitos danosos, tais como a sobrecarga dos operadores de saúde, a burocratização das práticas e o fomento de uma rede que, ao invés de ser acentrada, com múltiplas conexões, como em um modelo rizomático (Deleuze \& Guattari,1995), apresentase muito mais como um circuito que se retroalimenta (Rotelli, Leonardis, \& Mauri, 2001), causando a estagnação dos fluxos de experimentação e a reprodução de modos de relação homogêneos. Assim, ao contrário 
dos princípios de desmanicomialização, vão construindo-se outras segmentações que apartam, ou pelo menos, dificultam, os encontros com a diferença (p.7)

Acreditamos, pois, que a existência do ambulatório de saúde mental na atenção psicossocial no modelo que vimos anteriormente não se justifica, pois acaba recaindo em práticas que alimentam a exclusão da diferença. Entretanto, faz-se necessário investir em pesquisas sobre os ambulatórios de saúde mental no Brasil, subsidiando assim as reflexões necessárias para a mudança do modelo assistencial adotado e para o fortalecimento de arranjos organizacionais mais coerentes com a reforma psiquiátrica brasileira.

Ana Kalliny Severo

Doutoranda em Saúde Coletiva pela Universidade Estadual de Campinas, bolsista CAPES, Mestre em Psicologia pela Universidade Federal do Rio Grande do Norte, Rio Grande do Norte - RN - Brasil.

E-mail: kallinysevero@yahoo.com.br

Magda Dimenstein

Professora do Departamento de Psicologia da Universidade Federal do Rio Grande do Norte, pesquisadora do CNPq, Doutora em Saúde Mental pela Universidade Federal do Rio de Janeiro, Rio de Janeiro - RJ - Brasil. E-mail: magda@ufrnet.br

Endereço para envio de correspondência:

Rua Pintor Rodoldo de Amoedo, 528, Pitimbú, Natal - RN. CEP: 59069-150.

Recebido 29/8/2009, 1프 Reformulação 21/1/2011, Aprovado 1/4/2011. 


\section{Referências}

Afonso, M. L., \& Abade, F. L. (2008). Para reinventar as rodas: rodas de conversa em direitos humanos. Belo Horizonte: RECIMAM.

Alves, D. S., \& Guljor, A. P. (2004). O cuidado em saúde mental. In R. Pinheiro \& R. A. de Mattos (Orgs.), Cuidado: as fronteiras da integralidade (pp. 221-240). Rio de Janeiro: Hucitec.

Amarante, P. D. C. (2007). Saúde mental e atenção psicossocial. Rio de Janeiro: Fiocruz.

Baremblitt, G. (1992). Compêndio de análise institucional e outras correntes. Rio de Janeiro: Rosa dos Tempos.

Barros, R. B. (2003). Reforma psiquiátrica brasileira: resistências e capturas em tempos neoliberais. In Conselho Federal de Psicologia (Org.), Loucura, ética e política: escritos militantes (pp. 196-206). São Paulo: Casa do Psicólogo.

Bezerra Júnior, B. (2007). Considerações sobre terapêuticas ambulatoriais em saúde mental. In S. A. Tundis, \& N. R. Costa (Orgs.), Cidadania e loucura: políticas de saúde mental no Brasil (pp. 134-169). Petrópolis, RJ: Vozes.

Brasil. Ministério da Saúde. (1992). Portaria/SNAS no 224 - De 29 de janeiro de 1992. Recuperado em 30 de outubro de 2007 de http://www.inverso.org.br/index.php/content/ search_result/7.html?searchquery $=$ sih

Brasil. (2001). Lei $n^{\circ} 10.216$, de 06 de abril de 2001. Recuperado em 30 outubro 2007 dehttp://www.planalto.gov.br/ CCIVIL 03/Leis/LEIS 2001/L10216.htm.

Brasil. Ministério da Saúde. (2005). Reforma psiquiátrica e política de saúde mental no Brasil. Brasília, DF: Ministério da Saúde.

Brasil. Ministério da Saúde. (2006). Saúde mental no SUS: acesso ao tratamento e mudança do modelo de atenção. Brasília, DF: Ministério da Saúde.

Brasil. Ministério da Previdência Social. (2007). Diretrizes de conduta médico-pericial em transtornos mentais. Brasília, DF: Ministério da Previdência Social.

Cecilio, L. C. O. (1997). Modelos tecno-assistenciais em saúde: da pirâmide ao círculo, uma pirâmide a ser explorada. Cadernos Saúde Pública, 13(3), 469-478.

Coelho, M. (2008). A dimensão sociocultural da reforma psiquiátrica e a companhia experimental mudança. Saúde em Debate, 32, 92-98.

Costa-Rosa, A. (2000). O modo psicossocial: um paradigma das práticas substitutivas ao modo asilar. In P. Amarante (Org.), Ensaios, subjetividade e saúde mental (pp.141-168). Rio de Janeiro: Fiocruz.

Dell'Acqua, G., \& Mezzina, R. (2005). Resposta à crise: estratégia e intencionalidade da intervenção no serviço psiquiátrico territorial. In P. Amarante (Org.), Archivos de saúde mental e atenção psicossocial 2 (pp. 161-194). Rio de Janeiro: Nau.

Dimenstein, M., \& Liberato, M. (2009, maio). Desinstitucionalizar é ultrapassar fronteiras: o desafio da intersetorialidade e do trabalho em rede Cadernos Brasileiros de Saúde Mental, 1(1). [CD - ROM]

Goulart, M. S. B. (2004). Os descaminhos da política de saúde mental: um estudo sobre os serviços ambulatoriais nos anos 80. In I. F. Ferrari, \& J. N. G. Araújo (Orgs.), Psicologia e ciência na PUC MINAS (pp. 291-312). Belo Horizonte: PUC Minas.

Goulart, M. S. B. (2006). A construção da mudança nas instituições sociais: a reforma psiquiátrica. Pesquisas e práticas psicossociais, 1(1), 01-19.

Lougon, M. (2006). Psiquiatria institucional. Do hospício à reforma psiquiátrica. Rio de Janeiro: Fiocruz.

Natal, Secretaria Municipal de Saúde. (2006). Plano Municipal de Saúde: 2006-2009. Natal, RN: Secretaria Municipal de Saúde.
Natal, Secretaria Municipal de Saúde. (2008). Relatório de Gestão. Natal, RN: Secretaria Municipal de Saúde.

Passos, E., \& Barros, R. (2004). Clínica, política e as modulações do capitalismo. Lugar comum, 19/20, 159-171.

Peixoto, P. T. de C. (2007). Do esquadrinhamento dos corpos à invenção de práticas instituintes nos ambulatórios de saúde mental: três movimentos para a heterôgenese. Tese de doutorado, Universidade Federal Fluminense, Rio de Janeiro.

Rocha, M. L. (2006). Psicologia e as práticas institucionais: a pesquisa-intervenção em movimento. Psico, 37(2), 169-174.

Rocha, M. L. (2007). Micropolítica e o exercício da pesquisaintervenção: referenciais e dispositivos em análise. Psicologia: Ciência e Profissão, 27(4), 648-663.

Rocha, M. L. da R., \& Aguiar, K. F. (2003). Pesquisa-intervenção e a produção de novas análises. Psicologia: Ciência e Profissão, 23(4), 64-73.

Rodrigues, H. B. C. (2004). Análise institucional francesa e transformação social. O tempo (e contratempo) das intervenções. In S. Altoé (Org.), SaúdeLoucura 8 - análise institucional (pp. 115-164). São Paulo: Hucitec.

Rotelli, F., Leonardis, O., \& Mauri, D. (2001). Desinstitucionalização: uma outra via. In F. Nicácio (Org.), Desinstitucionalização (pp. 89-99). São Paulo: Hucitec

Santos, Y. (2007). O ambulatório de saúde mental no contexto da reforma psiquiátrica em Natal/RN. Dissertação de mestrado, Universidade Federal do Rio Grande do Norte, Rio Grande do Norte.

Severo, A. K. S. (2009). Cronificação nos serviços substitutivos na rede de saúde mental de Natal $R N$. Dissertação de mestrado, Universidade Federal do Rio Grande do Norte, Rio Grande do Norte.

Silva Filho, J. F. (2007). A medicina, a psiquiatria e a doença mental. In S. A. Tunds, \& N. R. Costa (Orgs.), Cidadania e loucura: políticas de saúde mental no Brasil (pp.75-102). Petrópolis, RJ: Vozes.

Vasconcelos, E. M. (2007). Dispositivos associativos de luta e empoderamento de usuários, familiares e trabalhadores em saúde mental no Brasil. Vivência, 32, 173-206.

Vasconcelos, E. M. (2009, maio). Epistemologia, diálogos e saberes: estratégias para práticas interparadigmáticas em saúde mental . Cadernos Brasileiros de Saúde Mental, 1(1). [CD - ROM]

Venturini, E., Galassi, A., Roda, A., \& Sérgio, E. (2003). Habilitarse em saúde mental: observações críticas ao conceito de reabilitação. Arquivos Brasileiros de Psicologia, 55(1), 56-63.

Yasui, S., \& Costa-Rosa, A. (2008). A estratégia atenção psicossocial: desafio na prática dos novos dispositivos de saúde mental. Saúde em Debate, 32(78-80), 27-37. 